\title{
Electronically Steerable Yagi-Uda Microstrip Patch Antenna Array
}

\author{
Derek Gray, Jun Wei Lu, and David V. Thiel
}

\begin{abstract}
This paper describes a dual-frequency circularly polarized electronically steerable microstrip patch antenna array suitable for land-mobile communications. Based on a fourelement Yagi-Uda patch antenna, the four antennas forming the array are located radially from a single square reflector patch on a double-sided printed circuit board. The main lobe of the array covers the elevation angles from 20 to $70^{\circ}$ with a peak gain of $8.4 \mathrm{dBi}$ at $1.54 \mathrm{GHz}$ and $11.7 \mathrm{dBi}$ at $1.62 \mathrm{GHz}$. Full azimuthal coverage is possible by electronically switching between the four elements.
\end{abstract}

Index Terms-Antenna arrays, microstrip antennas, Yagi-Uda arrays.

\section{INTRODUCTION}

$\mathbf{L}$ AND-MOBILE satellite communications are under development around the world, partly because suitable vehicle mounted compact antennas systems capable of tracking satellites are not available. The Australian telephone company Optus Series B Satellite has been designed for Mobilesat Service. This is a geostationary satellite having $L$ - and $\mathrm{Ku}$ band transponders for land-mobile communications across Australia and up to $200 \mathrm{~km}$ offshore. The frequencies for $L$ band are 1545.0-1559.0 MHz for receive and 1646.5-1660.5 $\mathrm{MHz}$ for transmit [1]. This requires elevation angles of $25-70^{\circ}$ above the horizon in Australia. This is the first such service to be offered in the world. Cost has proven to be a major impediment to the widespread adoption of this system. Thus, it is desirable to reduce the cost of the system hardware as much as possible to speed up the adoption of the system. The microstrip array described in this paper uses a patch Yagi-Uda antenna as an element.

The first microstrip Yagi-Uda antenna [2] had a maximum gain of $8 \mathrm{dBi}$ in the direction of the satellite. This antenna consisted of four closely spaced patches: a single driven patch, a larger reflector patch, and two smaller director patches. The dimensions of the patches and the spacing between them were based upon the design of the thin-wire Yagi-Uda antennas [3]. The arrangement has many advantages over single microstrip antennas including increased gain and increased directivity, particularly in directions away from the vertical. The input

Manuscript received September 18, 1995; revised May 22, 1997. This work was supported by a Grant from Griffith University, Queensland, Australia.

The authors are with the Radio Science Laboratory, School of Microelectronic Engineering, Griffith University, Nathan, QLD 4111, Australia.

Publisher Item Identifier S 0018-926X(98)03364-X. impedance bandwidth is increased significantly from that of a single patch because the parasitic patches allow the antenna structure to have multiple resonances [4]. This type of antenna can be used as a linearly polarized antenna for either vertical or horizontal polarization or, in a dual-feed configuration, as a circularly polarized antenna.

In addition, the Yagi-Uda configuration results in the beam peak being tilted away from the vertical in the end fire direction. Unlike phased arrays, there are no additional circuit elements such as power dividers or switchable phase delay transmission elements that introduce additional loss. The main design limitation for the microstrip Yagi-Uda antenna, unlike the thin-wire Yagi-Uda antenna, is that the design can only be realized for certain frequencies for a given dielectric substrate. Outside of this range, the patches are either too small and so the spacing between them is too large for the required mutual coupling or, at the other extreme, the patches are too large and would overlap. This limits the design to substrates $\varepsilon_{r}=1.5$ to $\varepsilon_{r}=5$ [2].

The feed position closest to the reflector patch is designated the $E$-plane feed and that at the side of the driven patch is designated the $H$-plane feed. The radiation from an antenna with only one feed is linearly polarized. When both the $E$ plane and $H$-plane feeds are used with an appropriate phase shift between them, the antenna radiation is circularly polarized. The handedness of the circular polarization is determined either by the side on which the $H$-plane feed is placed or the magnitude of phase difference between the feed points. The peak gain of a microstrip Yagi-Uda described in [2] is directed at $40^{\circ}$ from the broadside direction.

An array of four parallel, microstrip Yagi-Uda antennas for MSAT applications was described in [5] with a reported gain of $9.5-17 \mathrm{dBi}$ for the $20-60^{\circ}$ elevation angle. It was necessary to feed the four antennas in parallel to obtain this increased gain. Due to the enhanced bandwidth of the microstrip YagiUda antenna, this array was capable of both transmitting and receiving at the $L$-band frequencies required for land-mobile communications applications. The antenna main lobe lay in the required $20-60^{\circ}$ elevation range for the continental United States, while a pancake motor was used to steer the array in the azimuthal plane for satellite tracking [5].

The work reported in this paper follows on from the initial Yagi-Uda patch antenna [2]. The aim was to fabricate a low-cost low-powered electronically steered array suitable for land-mobile communications. The elimination of any mechanical steering apparatus greatly minimizes power 


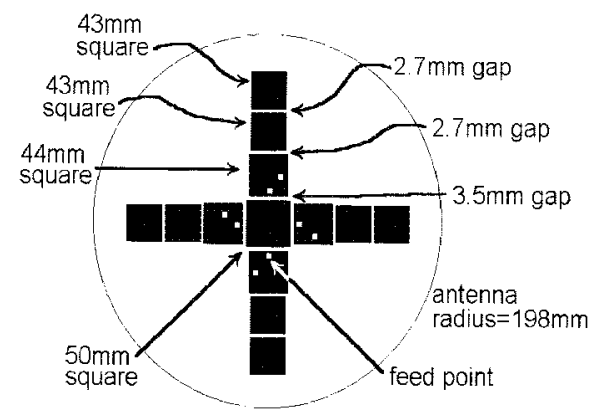

Fig. 1. Upper layer metallization of the Yagi-Uda microstrip patch antenna array.

requirements and so allows longer use if the motor vehicle does not have its engine running. It is possible to minimize fabrication costs by keeping support circuitry for the antenna as simple as possible. This also reduces RF losses within the circuitry. Applications of land mobile communications require an antenna system which has circular polarization, specific directional characteristics, rapid response/tracking speed, low fabrication cost, low maintenance cost (reliability, repair, etc.), mechanical durability, and preferably low profile, low weight, and a gain 9-12 $\mathrm{dBi}$ across the required elevation range.

For an electronically steered array, the response time/tracking speed is dependant only on the electronics. A purely electronically steered array has a lower fabrication cost because it is simpler to fabricate without a motor or weatherproof axial joint connection to the receiver. To achieve a lower maintenance cost, there is an obvious preference for electronics rather than electromechanical systems.

\section{Design And Construction}

The array [6] consists of four microstrip Yagi-Uda antennas arranged around a common reflector patch (Fig. 1). The array was fabricated on a laminate of three sheets of 1.6-mm-thick FR-4 epoxy/fiber-glass PCB substrate. This substrate has a dielectric constant of approximately $4 \varepsilon_{0}$, and is commonly used as a printed circuit-board substrate.

The control circuit for one element of this antenna is given in Fig. 2. The p-i-n diode is switched between being shorted to ground or an open circuit and is controlled by transistor-transistor logic (TTL). The RF switch (insertion loss of $1.1 \mathrm{~dB}$ ) is also controlled by the same logic signal. The choke prevents the RF signal from flowing back to the TTL logic circuit and the resistor is used to control the current through the diode. Thus, for TTL $=0 \mathrm{~V}, \mathrm{RF}$ power is delivered to the antenna and the diode has high impedance and for TTL $=5 \mathrm{~V}$ the antenna feeds are grounded and the RF line is not connected. The phase shifter provides a phase difference between the two feeds of each antenna to ensure circularly polarized radiation.

\section{EXPERIMENTAL RESULTS}

Polar pattern measurements were made at an open range at a height of $6 \mathrm{~m}$. The device under test was mounted on a turntable separated from a microwave horn source by a distance of $6.7 \mathrm{~m}$ (35 free-space wavelengths). The calibration

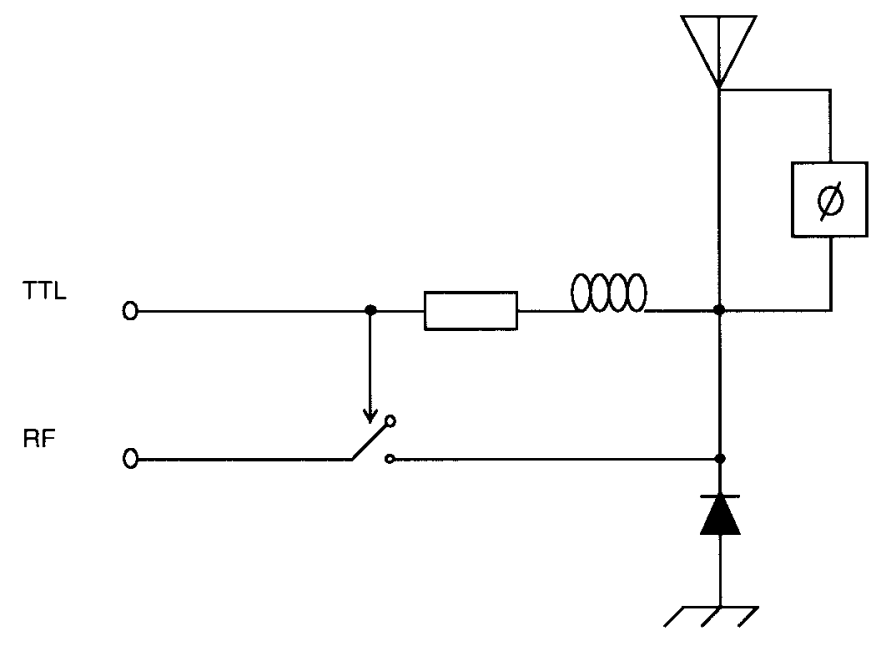

Fig. 2. Drive circuit for one element of the four element array. Control is based a single TTL signal.

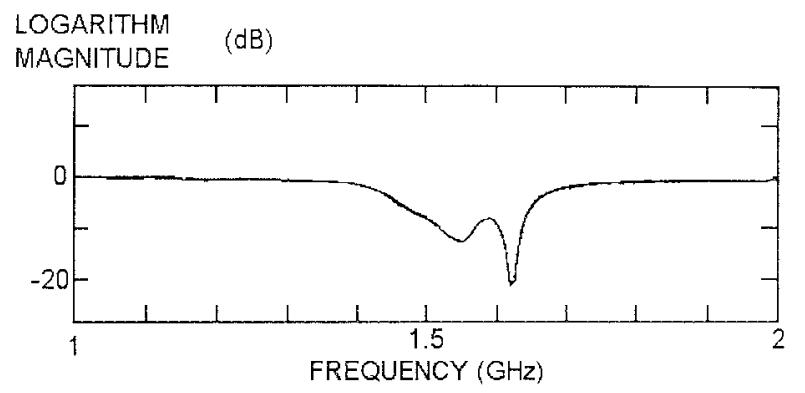

Fig. 3. $S_{11}$ scan showing the double resonance for the $E$ feed point of a single element of the array. The second feed point was terminated by $50 \Omega$ and the other active patch feeds were grounded.

of the antenna was achieved through a comparison with a standard gain horn. Only one of the four Yagi-Uda arrays was active during the trials, the others had their feed points short circuited to ground with shorting terminations. During the tests, the switching was done manually by swapping the feed and shorting terminations. The signal received at one feed of the active element was monitored for both source polarizations, while the other was terminated in a $50 \Omega$ termination. This was repeated for both feed positions. This technique allows one to optimize the phase shift between the two feed points for minimum cross polarization. Results were later combined mathematically to calculate the circular polarization characteristics.

The $S_{11}$ for both feed points of the array were measured across the frequency range 1-2 GHz. Fig. 3 illustrates the resonances at 1.54 and $1.62 \mathrm{GHz}$.

Fig. 4 shows the difference between the copolar and crosspolar signal levels as a function of phase shift at the $H$ feed point relative to the $E$ feed point for an elevation angle of $35^{\circ}$. Cross coupling is $-10 \mathrm{~dB}$ for both frequencies when the phase shift is $65^{\circ}$. This result is similar to [2] where a phase shift of $115^{\circ}$ was obtained with the $E$ feed point referenced to the $H$ feed point.

Fig. 5 illustrates the elevation polar patterns at both frequencies. The main lobe is directed in the $20-70^{\circ}$ elevation required for land-mobile satellite communications. For this 


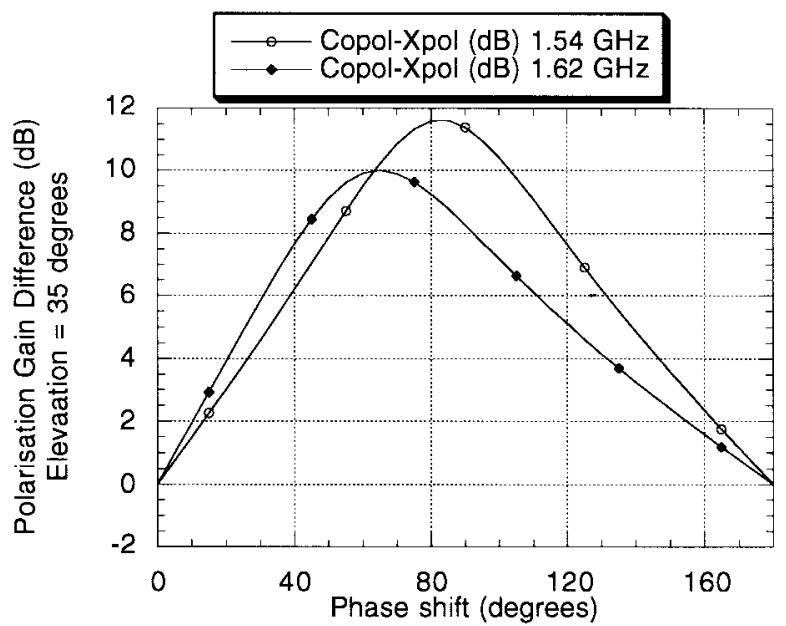

Fig. 4. Copolar and cross-polar gain determined at an elevation angle of $35^{\circ}$. A $65^{\circ}$ shift between the feeds gives an isolation ratio of $-10 \mathrm{~dB}$ at both frequencies.
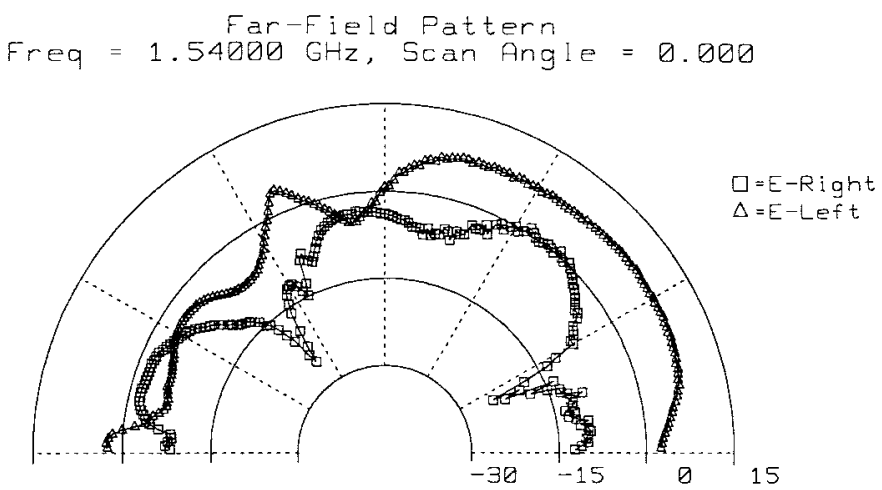

(a)
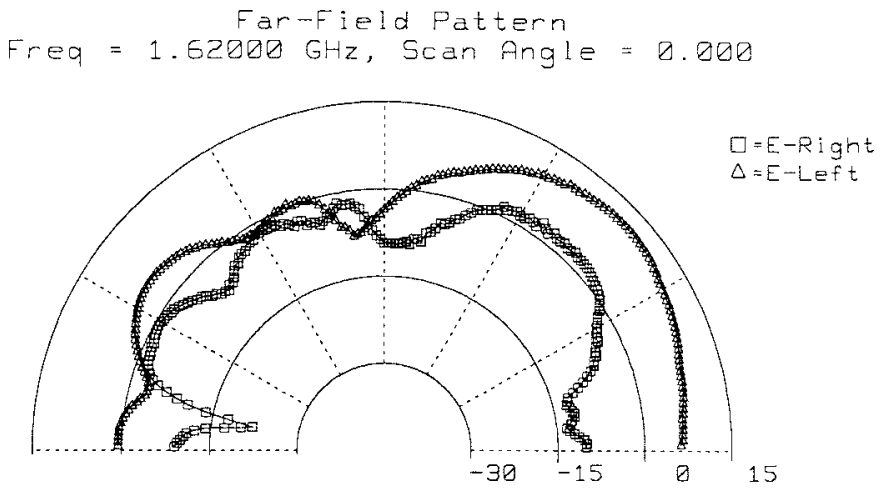

(b)

Fig. 5. (a) Elevation gain ( $\mathrm{dBi}$ ) of the array at $1.54 \mathrm{GHz}$ (left refers to copolar component; right refers to cross-polar component). (b) Elevation gain $(\mathrm{dBi})$ of the array at $1.62 \mathrm{GHz}$ (left refers to copolar component; right refers to cross-polar component).

elevation range, the peak gain for $1.54 \mathrm{GHz}$ was $8.4 \mathrm{dBi}$ and the minimum was $7.4 \mathrm{dBi}$. At $1.62 \mathrm{GHz}$ the peak was 11.7 $\mathrm{dBi}$ and the minimum was $8.9 \mathrm{dBi}$. These gains are below the required $+10 \mathrm{dBi}$ for high-latitude operation, but are suitable for operation at the intermediate latitudes found over most of continental Australia.
The azimuthal polar pattern was found to have a $100^{\circ}$ beamwidth at $-10 \mathrm{~dB}$ measured from the main lobe at both frequencies for an elevation angle range of $20-70^{\circ}$. This means that the switched array has a minimum gain of $0 \mathrm{dBi}$ at the $45^{\circ}$ azimuth point between two adjacent antennas.

\section{CONCLUSIONS}

The electronic switching between the four Yagi-Uda antennas radially oriented about a single reflector elements in the array has yielded $360^{\circ}$ azimuthal coverage at $20-70^{\circ}$ elevation angles within $0 \mathrm{dBi}$. While this gain figure is marginal at high latitudes, this $L$-band antenna array would be suitable as a low-profile low-cost land-mobile satellite communications antenna at low elevations. The chief advantages over the presently used omnidirectional antennas are the reduction in height (from a thick whip to a platter) and increased directivity in the direction of the satellite, which will conserve power and reduce multipath interference. Further improvements in gain would greatly improve the functionality of this antenna system. Addition of a fifth Yagi-Uda antenna would improve the azimuthal coverage.

\section{ACKNOWLEDGMENT}

The authors would like to thank Dr. T. Bird, K. Greene, and the staff of CSIRO Division of Radiophysics, Sydney, Australia, and also Assoc. Prof. B. M. Bialkowski and S. T. Jellett, University of Queensland, Australia, where the antenna polar-pattern measurements were made.

\section{REFERENCES}

[1] A. Kumar, Fixed and Mobile Terminal Antennas. Boston, MA: Artech House, 1991.

[2] J. Huang, "Planar microstrip Yagi array antenna," in IEEE Antenna Propagat. Soc. Symp. Dig., June 1989, pp. 894-897.

[3] H. Yagi, "Beam transmission of ultrashort waves," Proc. Inst. Radio Eng. (IRE), vol. 16, pp. 715-741, June 1928.

[4] D. M. Pozar, "A review of bandwidth enhancement techniques for microstrip antennas," in Microstrip Antennas, D. M. Pozar and D. H. Schaubert, Eds. New York: IEEE Press, 1995, pp. 157-166.

[5] J. Huang and A. C. Densmore, "Microstrip Yagi array antenna for mobile satellite vehicle application," IEEE Trans. Antennas Propagat., vol. 39, pp. 1024-1030, July 1991.

[6] D. Gray, J. W. Lu, and D. V. Thiel, "Electronically steered microstrip Yagi Uda antenna array," IEEE Antennas Propagat. Soc. Symp. Dig., Newport Beach, CA, June 1995 p. 1870.

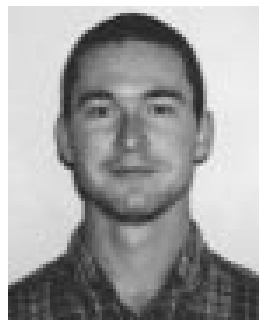

Derek Gray received the B.E. (microelectronics engineering) and M.Phil. degrees from Griffith University, Nathan, Australia, in 1994 and 1996, respectively. He is currently working toward the Ph.D degree at the University of Manitoba, Winnipeg, Canada.

His research interests include computational electromagnetics for analysis and synthesis of small planar antennas, small antennas design, and mobile terminal antenna design.

Mr. Gray received a TRLabs Scholarship in 1996 


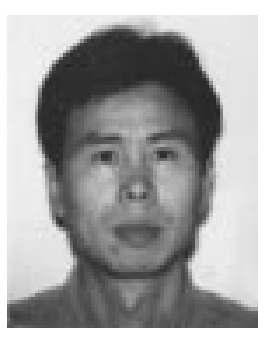

Jun Wei Lu received the B.Eng. degree from the Department of Electrical Engineering, Xian Jiaotong University, Xian, China, in 1976, the M.Eng. degree from the National Toyama University, Toyama, Japan, in 1988, and the Ph.D. degree from the National Kanazawa University, Kanazawa, Japan, in 1991.

From 1976 to 1983 , he worked with the Institute of Qinhai Electric Power Testing and Research, Xinin, China. He has also worked on applied computational electromagnetics for magnetic devices with the Electrical Engineering Coversion Laboratory at Kanazawa University. He joined Griffith University, Queensland Australia, in 1992, and is a Senior Lecturer at the School of Microelectronic Engineering at that university. His fields of interest include computational and visual electromagnetics and its applications in the design and analysis of mobile antennas and arrays, RF/MW devices and circuits, and high-frequency magnetic devices.

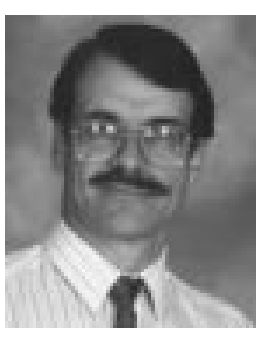

David V. Thiel received the S.Sc. degree from the University of Adelaide, Adelaide, Australia, in 1970, and the M.Sc. and Ph.D. degrees from James Cook University, Townsville, Australia, in 1974 and 1980, respectively.

$\mathrm{He}$ is currently Professor and Head of the School of Microelectronic Engineering at Griffith University, Queensland, Australia, and Director of the Radio Science Laboratory at the same university.

Dr. Thiel was elected to the Antenna and Propagtion Society ADCOM in 1997 and serves on the Wave Propagation Standards Committee. 